\title{
Comment garantir des données médicales de qualité dans les études cliniques, pharmaco- épidémiologiques et en pharmacovigilance ?
}

François Alla ${ }^{1}$, Myriam Rosilio ${ }^{2}$, Christian Funck-Brentano ${ }^{3}$, et les participants à la table ronde $N^{\circ} 2$ de Giens XXVIII : Philippe Barthélémy ${ }^{4}$, Sophie Brisset ${ }^{5}$, Dominic Cellier $^{6}$, Olivier Chassany ${ }^{7}$, Jean-Paul Demarez ${ }^{8}$, Vincent Diebolt ${ }^{9}$, Alain Francillon ${ }^{10}$, Laetitia Gambotti ${ }^{11}$, Hakima Hannachi ${ }^{12}$, Philippe Lechat ${ }^{13}$, François Lemaire ${ }^{14}$, Michel Lièvre ${ }^{15}$, Christophe Misse ${ }^{16}$, Marina Nguon ${ }^{17}$, Antoine Pariente ${ }^{18}$, Michel Rosenheim ${ }^{19}$ et Nadine Weisslinger-Darmon ${ }^{20 \dagger}$

1 Université de Lorraine, Nancy, France

2 Laboratoire Lilly France, Neuilly-sur-Seine, France

3 Université Pierre et Marie Curie, Paris, France

4 Laboratoire AstraZeneca, Rueil-Malmaison, France

5 Laboratoire Ax-Pharma, Paris France

6 Laboratoire Merck Serono, Lyon, France

DRCD, Paris, France

8 Laboratoire Pierre Fabre Médicament, Castres, France

9 F-CRIN, Toulouse, France

10 Laboratoire Novartis, Rueil-Malmaison, France

11 URC Pitié-Salpétrière, AP-HP, Paris, France

12 Laboratoire Merck Sharp Dohme, Courbevoie, France

13 Université Paris 7, Paris, France

14 DRCD Île-de-France, Paris, France

15 Pharmacologie clinique, CHU, Lyon, France

16 DRCD, Paris, France

17 DRCD, Pôle vigilance, Paris, France

18 Centre régional de Pharmacovigilance, $\mathrm{CHU}$, Bordeaux, France

19 Assistance Publique - Hôpitaux de Paris, Paris, France

20 Laboratoire GlaxoSmithKline, Marly-le-Roi, France

Texte reçu le 15 mars 2013 ; accepté le 7 mai 2013

\section{Mots clés :}

qualité ; données ;

recommandations ;

essais cliniques ;

pharmaco-

épidémiologie ;

pharmacovigilance
Résumé - Le développement des médicaments fait l'objet de normes de qualité qui visent à garantir que le contenu d'une base de données soit un reflet fidèle du dossier source. Paradoxalement, ces normes abordent peu la qualité de la donnée source elle-même. L'objectif de ce travail était de proposer des recommandations pour améliorer la qualité des données dans trois champs (études cliniques, pharmaco-épidémiologiques et de pharmacovigilance). L'analyse s'est centrée sur les données et les étapes critiques, qui posent un problème important de qualité et pour lesquelles les recommandations actuelles ne sont pas suffisamment détaillées, peu adaptées et/ou appliquées. Au final, des recommandations ont été formulées qui portent principalement sur la genèse de la donnée et sa transcription.

Abréviations : voir en fin d'article.

$\dagger$ Les articles, analyses et propositions issus des Ateliers de Giens n’engagent que leurs auteurs et ne préjugent pas de la position de leur organisme de tutelle. 


\section{Introduction}

Le développement des médicaments fait depuis de nombreuses années l'objet de normes de qualité très strictes, à l'exemple des normes de l'international conference of harmonization (ICH) pour les essais cliniques. ${ }^{[1]}$ Ces recommandations et procédures visent généralement à garantir que le contenu d'une base de données soit un reflet fidèle du dossier source. Cependant, elles abordent peu, voire n'abordent pas, un aspect pourtant essentiel : la qualité de la donnée source. L'exemple de l'évaluation d'évènements critiques lors d'un essai clinique montre pourtant que les interprétations des médecins investigateurs peuvent être pour le moins questionnées. Plusieurs travaux ont par exemple montré que la classification proposée par les investigateurs pour les évènements majeurs cardiovasculaires était changée par un Comité indépendant d'évaluation des évènements critiques (adjudication committee) dans un quart à un tiers des cas. ${ }^{[2-4]}$ La mauvaise qualité de l'information médicale contenue dans la donnée source pourrait ainsi conduire à changer la conclusion d'un essai. De la même façon, un manque de qualité de l'information médicale contenue dans les données de pharmaco-épidémiologie ou de pharmacovigilance peut être lourd de conséquences. Les risques liés à l'absence de qualité des données et à la diffusion et l'utilisation d'informations non valides sont en effet, au-delà de la sécurité du patient et de la santé publique, de nature décisionnelle (par exemple prendre une décision réglementaire inadéquate), de nature médiatique et sociale (les exemples sont nombreux dans l'actualité !) et de nature scientifique (en particulier de mauvaises données peuvent conduire à générer des hypothèses fausses qu'il faudra du temps et des moyens pour récuser). Ce risque informationnel devient une préoccupation affichée du régulateur, comme l'illustre la proposition de règlement européen relatif aux essais cliniques dont un objectif est de «[...] parer à deux risques distincts : le risque pour la sécurité des participants et le risque pour la fiabilité des données. » ${ }^{[5]} \mathrm{S}$ 'intéresser à la qualité de l'information médicale générée par les données sources est donc un enjeu majeur, et pourtant, nous le verrons, relativement peu exploré dans la littérature.

L'objectif de ce travail était de proposer des recommandations pour améliorer la qualité des données, et plus précisément leur « validité médicale » dans trois types d'études (études cliniques, pharmaco-épidémiologiques et de pharmacovigilance). La démarche a été basée sur l'échange d'observations, d'expériences et d'expertises entre des acteurs de ces trois champs. Le travail s'est centré sur la qualité de la donnée et n'a pas abordé celle de son utilisation (à l'exemple de l'analyse statistique). Les propositions exposées en fin de cet article ont été émises après une démarche structurée, dont les éléments sont présentés dans cet article : définition du cadre, en particulier des données et de leurs sources, description des processus et mise en évidence des points critiques, puis analyse des recommandations existantes et de leurs limites.

\section{Cadrage, typologies et définitions}

Le travail a porté sur les données et informations médicales utilisées dans trois principaux champs de l'évaluation et de la sécurité en matière de médicament : études cliniques, pharmaco-épidémiologie et pharmacovigilance définis comme suit :

1. Le champ des études cliniques de phase III : les essais de phase III permettent d'évaluer le rapport bénéfice/risque d'un futur médicament, notamment par une meilleure appréciation de son efficacité et de sa tolérance à moyen et long terme. Les contrôles de qualité et les audits sont fréquemment effectués sur ces études, en particulier lorsqu'il s'agit d'essais destinés à l'obtention d'une autorisation de mise sur le marché (AMM).

2. Le champ de la pharmaco-épidémiologie est l'étude de l'utilisation et des effets des médicaments sur de larges populations pour décrire et analyser des pratiques et des conditions d'utilisation, évaluer la sécurité, évaluer l'efficacité (en alternative à l'essai), évaluer l'efficacité en situation réelle ([comparative] effectiveness research), effectuer des évaluations économiques et médico-économiques. ${ }^{[6]}$ Elle peut s'insérer dans un contexte réglementaire (ex. : études post-inscriptions ; études post AMM).

3. Le champ de la pharmacovigilance : la pharmacovigilance a pour objet la surveillance du risque d'effet indésirable résultant de l'utilisation des médicaments, l'analyse et la gestion de ces risques. Dans le cadre des essais cliniques, la pharmacovigilance joue un rôle important puisque par définition la tolérance du médicament à l'étude est évaluée lors de ces essais. Une attention particulière est portée aux suspected unexpected serious adverse reaction (SUSAR), qui sont les effets indésirables graves inattendus ou dont l'évolution est inhabituelle, pour lesquels il existe une relation avec le médicament expérimental.

\subsection{Quelles données?}

Toutes les données contributives à l'objectif d'une étude sont concernées par cette analyse. Il s'agit particulièrement des données décrivant et fournissant des éléments de vérification des états et évènements de santé, des antécédents et autres caractéristiques cliniques et comportementales des patients, les procédures diagnostiques et leurs résultats, des traitements et interventions, de l'observance et de la persistance, etc. Ces données peuvent être utilisées entre autres pour sélectionner et définir la population, comme facteurs d'exposition, facteurs d'ajustement et critères de jugement.

\subsection{Quelles sources?}

Certaines sources sont plus spécifiques à certains champs. Les essais cliniques reposent généralement sur des données spécifiquement recueillies et/ou issues de la prise en charge médicale. La 
Tableau I. Description des champs et des sources identifiés.

\begin{tabular}{|l|c|c|c}
\hline \multicolumn{1}{|l|}{ Champs } & Essai clinique phase III & Pharmacovigilance & Pharmaco-épidémiologie \\
\cline { 1 - 2 } Sources & $\mathrm{xxx}$ & $\mathrm{x} / \mathrm{xx}$ & $\mathrm{xx}$ \\
\hline Données spécifiques & $\mathrm{xx}$ & $\mathrm{xx}$ & $\mathrm{xx}$ \\
\hline Données rétrospectives & $\mathrm{xx}$ & $\mathrm{xxx}$ & $\mathrm{x}$ \\
\hline Déclarations & $\mathrm{x}$ & $\mathrm{x}$ & $\mathrm{xx}$ \\
\hline Données médico-administratives & & & \\
\hline
\end{tabular}

$\mathrm{x}$ : donnée peu utilisée dans le champ mentionné ;

$\mathrm{xx}$ : donnée moyennement utilisée dans le champ mentionné ;

$\mathrm{xxx}$ : donnée très fréquemment utilisée dans le champ mentionné.

pharmaco-épidémiologie quant à elle se développe avec l'exploitation des données médico-administratives. Enfin, la pharmacovigilance utilise classiquement les données de notifications soit en provenance des essais cliniques, soit provenant des déclarations spontanées colligées parfois dans le cadre de suivis prospectifs pour un plan de gestion de risque.

Les sources de ces données sont potentiellement nombreuses : - les données spécifiquement recueillies (ou données dites «prospectives») : les mesures (examen clinique, questionnaire, bilan complémentaire...) sont réalisées pour l'étude ;

- les données, notamment extraites des dossiers médicaux, issues de la prise en charge courante (ou données dites « rétrospectives ») : les mesures ont été réalisées dans le cadre du soin et elles sont ré-utilisées pour l'étude ;

- les données médico-administratives : ces données ont été initialement recueillies pour la gestion et le financement des soins. Elles peuvent être utilisées secondairement dans un objectif de santé publique ou de recherche. En France, les plus utilisées sont les données du programme de médicalisation des systèmes d'information (PMSI) et du système national d'informations inter régions d'Assurance maladie (SNIIRAM) ;

- les données de pharmacovigilance sont obtenues dans le cadre du dispositif national de pharmacovigilance mais aussi dans le cadre du suivi de la tolérance de médicaments testés dans des essais cliniques : déclarations d'évènements indésirables non graves, graves et inattendus recueillis lors des essais cliniques mais aussi les données issues de notifications spontanées pour les médicaments déjà commercialisés.

De par ces spécificités, les recommandations actuelles sont généralement segmentées et ne couvrent pas l'ensemble des sources pour une utilisation donnée. Cependant, les utilisations tendent à se diversifier et chacun de ces champs est désormais susceptible d'utiliser chacune de ces sources voire même leur combinaison (tableau I).

Pour cette raison et de part la similitude des problématiques, les trois champs ont été analysés ensemble.

\subsection{De la donnée à l'information}

Tout d'abord, - et c'est majeur - il convient de distinguer la notion de «donnée » (élément brut recueilli directement par mesure ou issu d'une donnée recueillie dans un autre objectif avec changement de finalité) de la notion « d'information », qui résulte du traitement et de l'interprétation d'une donnée brute. Par exemple, le poids et la taille sont des données, la présence ou non d'une obésité, définie par une combinaison de ces données à laquelle on applique un seuil, est une information. Une donnée peut être de qualité, mais conduire en fonction de son traitement et de son interprétation à une information de qualité médiocre. Cette distinction est particulièrement importante à prendre en compte dans le cadre de l'utilisation de données médico-administratives. En effet, ces données sont en général de qualité (par exemple l'information dans une base de remboursement « délivrance d'un traitement à visée antihypertensive » correspond à la réalité). Cependant, déduire de la délivrance d'un médicament à visée antihypertensive que le patient est hypertendu est une interprétation qui peut être problématique (par exemple par manque de spécificité du traitement).

\subsection{Qu'est ce qu'une donnée de qualité ?}

Une donnée de qualité pourrait être définie comme une donnée qui représente correctement le réel. Cette définition est la plus naturelle, mais elle présente l'inconvénient d'induire la perception d'une qualité «absolue» pour laquelle le plus est toujours le mieux. Cet absolu n'est pourtant pas envisageable, ni même souhaitable : par exemple à quoi servirait d'avoir le poids d'un patient au gramme près ? Le niveau de qualité optimal dépend de l'objectif: par exemple si la mesure de la masse ventriculaire gauche de référence est l'imagerie par résonnance magnétique (IRM), son estimation par l'echocardiographie peut être considérée de qualité suffisante si l'objectif est de recruter des patients 


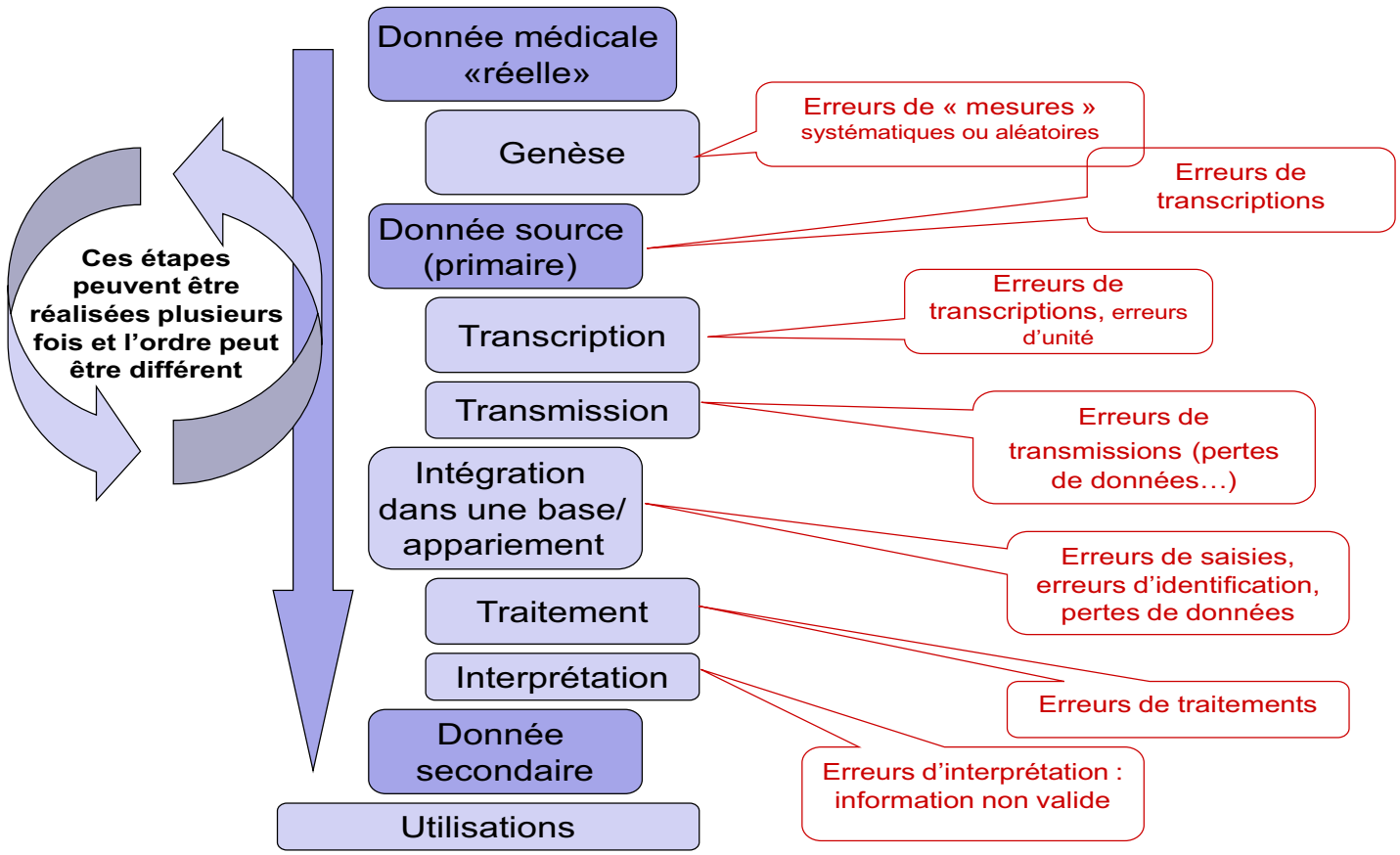

Fig. 1. La chaîne de la donnée : différentes étapes et exemples d'erreurs pouvant survenir.

avec hypertrophie ventriculaire gauche. De plus, un niveau de qualité donné est à mettre en balance avec la faisabilité, les coûts et les risques pour son obtention. Enfin, il ne faudrait pas qu'une vision absolue de la qualité induise des conditions de recherche tellement éloignées de la pratique courante que la transposabilité des résultats de cette recherche s'en trouve compromise. Ainsi, on pourrait proposer une définition plus opérationnelle : une donnée de qualité est une donnée qui répond de façon appropriée à ses objectifs d'utilisation.

Par conséquent, nous avons défini une donnée de qualité non seulement comme une donnée qui représente correctement le réel, mais surtout comme une donnée conduisant dans un cadre défini à une information de qualité permettant de prendre la bonne décision, au bon moment.

\subsection{Critères de qualité}

Plusieurs dizaines de critères de qualité ont été proposés dans la littérature en santé ou du domaine de la qualité en général. Pour être synthétique, trois champs de la qualité (donnée, information, processus) pourraient être distingués et pour chacun d'eux un certain nombre de critères pourraient être listés. Les trois critères de qualité de la donnée que nous avons retenus sont la validité (ou l'exactitude), la précision et la fiabilité. Les critères de qualité de l'information (qui se juge aussi par rapport à son utilisation) sont, outre les trois critères précédents, la pertinence (ou l'utilité ou le caractère approprié), l'accessibilité, l'exhaustivité, l'objectivité de l'interprétation. Les critères de qualité du processus d'obtention de l'information sont : le caractère coût-efficace, l'absence de risques, la confidentialité, la sécurité, la pérennité, l'organisation et le caractère vérifiable (auditable) de l'information.

\section{Description des processus et mise en évidence des points critiques}

Dans un premier temps, nous avons décrit les étapes, de la génération à l'utilisation des données (figure 1). Cette représentation est bien entendu schématique et générique : en fonction du champ et de l'étude, l'ordre de ces étapes peut être différent, certaines peuvent être réalisées plusieurs fois. Puis, pour chaque étape, les principales erreurs pouvant survenir ont été listées. Pour chaque erreur, ses principales causes ont été identifiées.

L'analyse s'est ensuite centrée sur les données et les étapes critiques. Nous avons considéré que les données critiques étaient celles dont la qualité (ou l'absence de qualité) posait un problème majeur de risque. Il s'agit en particulier du critère de jugement principal, des événements graves, de données modifiant le rapport bénéfice-risque, etc. Pour ces données critiques, nous avons considéré que les étapes critiques qui leurs correspondaient étaient celles qui posaient un problème important de qualité et pour lesquelles les 
recommandations ne semblaient pas suffisamment détaillées, peu adaptées et/ou imparfaitement appliquées.

\section{Analyse de l'existant et élaboration de recommandations}

Pour répondre à ces enjeux et s'assurer de la qualité des données, il existe déjà, dans les trois champs, de nombreuses recommandations et procédures relatives aux données et à leur traitement. À titre d'exemple peuvent être cités pour les essais cliniques, les recommandations $\mathrm{ICH}^{[1]}$ ou plus spécifiquement le guideline on missing data in confirmatory clinical trials, ${ }^{[7]}$ pour la pharmacoépidémiologie, le guide on methodological standards in pharmacoepidemiology de l'Agence européenne du médicament (EMA), ${ }^{[8]}$ et le guidelines for good pharmacoepidemiology practices de l'International Society of Pharmacoepidemiology (ISPE), ${ }^{[9]}$ et enfin pour la pharmacovigilance, les nouvelles recommandations européennes de bonnes pratiques de pharmacovigilance. ${ }^{[10]}$

Ces recommandations et procédures visent généralement à garantir que le contenu d'une base de données soit un reflet fidèle du dossier source. Il existe ainsi de nombreuses procédures relatives au data management. ${ }^{[11-13]}$ Cependant, l'analyse permet de mettre en évidence que ces recommandations ne sont pas assez détaillées sur un point essentiel et critique : la validation de la qualité des données sources et de l'information qui permet d'en garantir la validité/véracité au sens médical du terme (par exemple, ce qui est déclaré comme un infarctus du myocarde est-il vraiment un infarctus du myocarde; quels critères ont été utilisés pour ce diagnostic ?). Les recommandations existantes ne mentionnent en effet pas (ou peu) ces aspects, en particulier la validité médicale de la donnée source. Cette dernière est en effet implicitement considérée comme un gold standard et donc à ne pas remettre en question. Ainsi, dans les trois champs, l'étape de la genèse de la donnée (c'est-à-dire l'étape constitutive de la donnée source par mesure ou observation) nous a semblé prioritaire. Il s'agit de la qualité du diagnostic médical initial, mais aussi de l'exactitude des mesures utilisées (anthropométriques, biologiques, électrocardiographiques...).

D'autres étapes critiques ont été mises en évidence dans la chaîne de traitement de données, qui dépendent du champ et de la source de données (figure 1).

Pour les données cliniques, biologiques ou médico-administratives, la genèse de la donnée (erreur de diagnostic, dossier médical incomplet ou avec erreurs) et sa transcription (erreurs, codage inadapté, utilisation d'un code imprécis) représentent un point critique majeur. Plus spécifiquement pour les données médico-administratives, si un risque de pertes de données ou erreur d'appariement existe lors des étapes suivantes de transmission, traitement et intégration des données dans les bases, ces erreurs sont généralement limitées par les nombreuses procédures existantes (qui restent toutefois peu connues des utilisateurs).
Pour les données médico-administratives, un deuxième point critique est l'interprétation de la donnée. Cette interprétation réalisée lors du traitement statistique des données en vue de leur analyse peut être source d'erreurs. Les principaux points critiques sont : la définition du dénominateur (patients) ; l'absence de données cliniques (diagnostic clinique, comorbidités, antécédents, facteurs de risques) ou leur imprécision ; la définition de l'exposition au traitement. Plus qu'un problème de qualité, nous sommes ici face à un problème d'utilisation. La prise en compte de ces limites repose sur des techniques d'analyse spécifiques. Abordant ces problématiques, il existe quelques recommandations génériques (par exemples les guides de pharmaco-épidémiologie déjà cités) et d'autres plus spécifiques. ${ }^{[14-16]}$ Cependant, la littérature s'accorde sur leur insuffisance. ${ }^{[17-20]}$

La qualité des données en pharmacovigilance est une préoccupation partagée. ${ }^{[21]}$ Plusieurs points critiques ont particulièrement retenu notre attention. La donnée médicale est collectée, transmise et codée avec le medical dictionnary for regulatory activities (dictionnaire MEDRA). Ce dictionnaire est complexe et nécessite une formation spécifique afin d'éviter une variabilité dans les codages. Par ailleurs, le problème de l'interprétation de la donnée de pharmacovigilance déclarative est majeur. En effet, le fait de relier ou non un évènement à un médicament a des conséquences importantes. Il est donc nécessaire que cette donnée de «causalité/imputabilité » soit validée de façon collégiale, sans biais d'interprétation.

\section{Recommandations}

Au final, à partir des analyses précédemment détaillées, les recommandations suivantes ont été formulées selon un principe commun (figure 2). Certaines de ces recommandations sont originales, d'autres sont adaptées des recommandations existantes dont l'application devrait être renforcée.

\subsection{Genèse de la donnée "médicale » et sa transcription}

\subsubsection{Concernant l'investigateur}

- certifier l'investigateur : certification focalisée sur la qualité informative de la donnée médicale (formation labélisée), avec décertification possible s'il apparait que l'investigateur ne satisfait pas aux obligations et au respect des procédures ;

- poursuivre et renforcer la professionnalisation de la recherche : en particulier privilégier la présence de techniciens de recherche clinique infirmiers dans les services ;

- améliorer l'accessibilité des outils de déclarations de pharmacovigilance (ex. : portail web unique pour toutes les vigilances) ;

- pondérer la rémunération de l'investigateur sur la validité de l'information médicale liée aux données le cas échéant. 


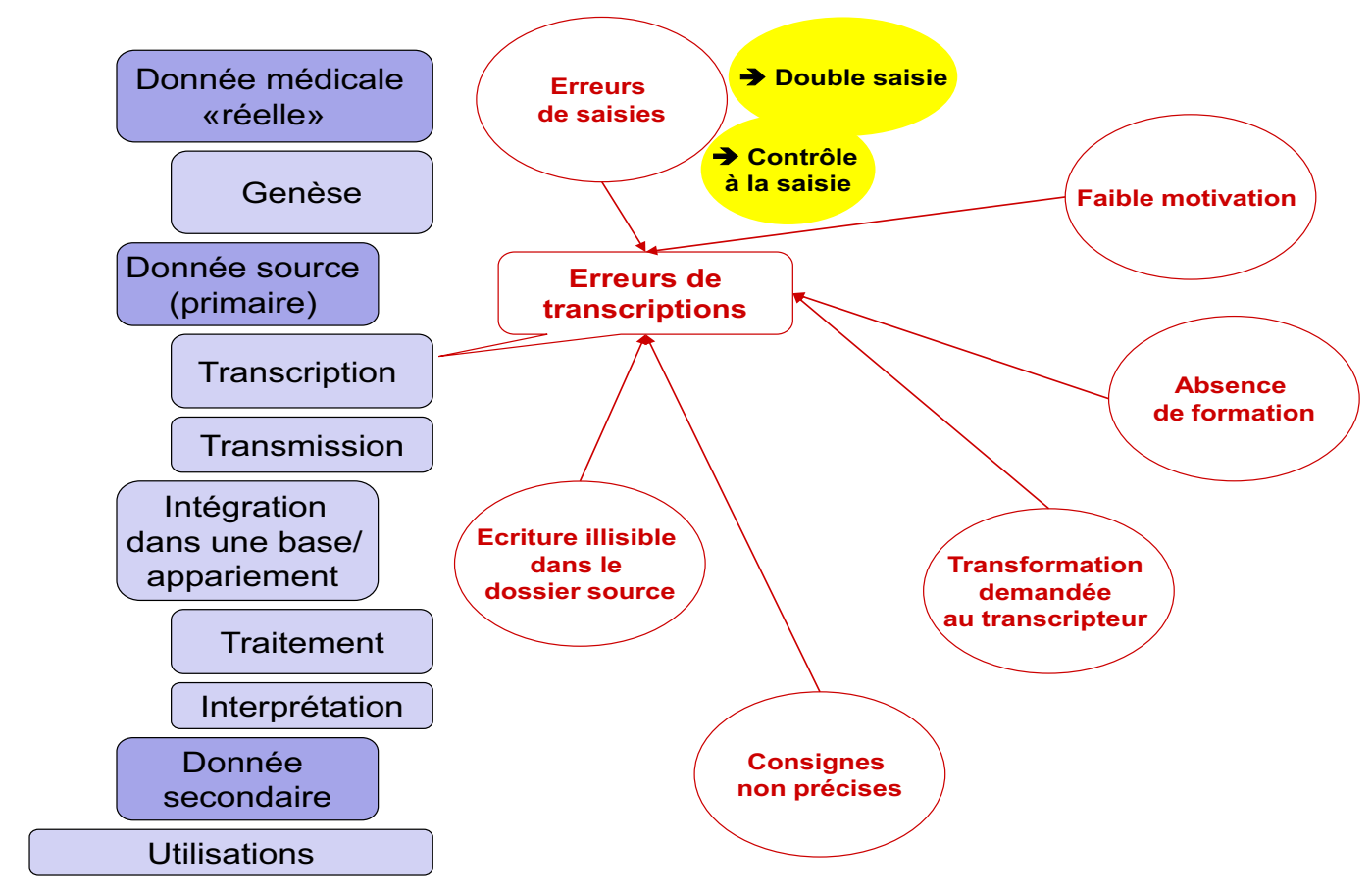

Fig. 2. Exemple de causes pour un type d'erreur et exemples de procédures pour réduire ce type d'erreur.

\subsubsection{Concernant la genèse, la transcription et la validation des données critiques}

- identifier dans le protocole les informations critiques qui doivent être obligatoirement renseignées et validées par un médecin et non pas seulement sous sa direction ;

- prévoir des procédures adaptées au protocole permettant de renforcer la qualité des informations critiques (ex. : contacts directs attachés de recherche clinique (ARC)/investigateur, «cadre rouge » des questions à remplir impérativement par le médecin, procédure de revue des dossiers avec l'investigateur pour vérifier véracité et documentation pour ces données...);

- prévoir des procédures de validation de ces informations critiques (ex : fonction de «médecin évènements » auprès du promoteur ; validation systématique par un comité indépendant d'évaluation des événements critiques) ;

- respecter des bonnes pratiques métrologiques et psychométriques en matière de développement, validations et adaptations transculturelles d'outils de mesures (en particulier pour la mesure des patient reported outcomes) ;

- respecter les bonnes pratiques de laboratoire, les procédures d'étalonnage..., favoriser la centralisation des analyses le cas échéant ;

- simplifier des outils de recueil : se limiter aux informations essentielles (rechercher la qualité et la complétude des données essentielles plutôt que la quantité des données avec comme perspective l'analyse finale qui en sera faite) ;

- améliorer la clarté et l'explicitation des consignes ;

- systématiser l'utilisation de documents traduits et validés dans la langue de l'investigateur et du patients le cas échéant ;

- standardiser les définitions des états de santé, des éléments d'objectivation et de leur codage. Des recommandations et/ou consensus thématiques professionnels adaptés au champ de la recherche et de la pharmacovigilance devraient être effectués ;

- valoriser l'utilisation de cahier d'observation (case report form [CRF]) électroniques (qui doivent respecter les critères de parcimonie en termes de nombre de données collectées);

- mettre en place des procédures de détection en temps réel des variations anormales dans les données cliniques ou biologiques (e-CRF ou alerte labo) ;

- focaliser l'attention des acteurs de la pharmacovigilance sur les événements graves (au sens réglementaire du terme);

- standardiser les codifications (notamment pour MedDRA), notamment en évitant de multiplier les codes pour les mêmes entités médicales ;

- inclure dans le consentement patient une acceptation pour être contacté et/ou pour que les établissements et médecins l'ayant pris en charge puissent l'être afin de compléter les informations de pharmacovigilance ou de suivi (notamment si le patient est perdu de vue). 


\subsection{Traitement des données}

- validation (pour les données critiques) des définitions et algorithmes utilisés ;

- expliciter les procédures de data management (notamment pour les bases médico-administratives) ;

- développer les procédures de contrôle de cohérences internes et externes.

\subsection{Reporting/publication}

- formaliser un reporting des méthodes et résultats dans les compte-rendus, notamment les articles, permettant d'apprécier la qualité des données (approfondissement sur ce point des grilles type consolidated standard of reporting trials [CONSORT] et strengthening the reporting of observational studies in epidemiology [STROBE] checklists, good research for comparative effectiveness [GRACE], ISPE checklist, etc.)

\section{Conclusion}

Nous avons donc revu la chaîne de la donnée dans ces différents champs et pour différentes sources utilisées, et avons tenté de proposer des solutions destinées à améliorer la qualité de l'information médicale issue de ces données. Bien que les champs soient différents, les problématiques identifiées sont similaires.

Certaines actions permettant d'assurer la qualité sont déjà mentionnées dans les recommandations en vigueur et doivent être mieux intégrées, comprises et appliquées dans la pratique. Ce constat et ces propositions pourront contribuer à l'élaboration de procédures simples et applicables centrées sur l'essentiel : l'amélioration de l'information médicale générée dans les essais cliniques, les études pharmaco-épidémiologiques et les données de pharmacovigilance.

\section{Conflits d'intérêts. Aucun.}

\footnotetext{
Abréviations. AMM : autorisation de mise sur le marché ; ARC : attaché de recherche clinique ; CONSORT : consolidated standard of reporting trials ; CRF : case report form ; EMA : Agence européenne du médicament ; GRACE : good research for comparative effectiveness ; ICH : international conference of harmonization ; IRM : imagerie par résonnance magnétique ; ISPE : International Society of Pharmacology; MEDRA (dictionnaire) : medical dictionnary for regulatory activities ; PMSI : programme de médicalisation des systèmes d'information ; SNIIRAM : système national d'informations inter régions d'Assurance maladie; STROBE: strengthening the reporting of observtional studies in epidemiology; SUSAR : suspected unexpected serious adverse reactions.
}

\section{Références}

1. ICH. Guideline for good clinical practice E6 (R1). 1996 http://www.ich.org/ fileadmin/Public_Web_Site/ICH_Products/Guidelines/Efficacy/E6_R1/ Step4/E6_R1_Guideline.pdf

2. Näslund U, Gript L, Fischer-Hansen J, et al. The impact of an end-point committee in a large multicenter, randomized, placebo-controlled clinical trial: results with and without the end-point committee's final decision on endpoints. Eur Heart J 1999; 20(10): 771-7

3. Heagerty A, Deverly A, Palmer C, et al. The role of the critical event committee in a major cardiovascular outcome study. Blood Press 2002; 11(6): 339-44

4. Mahaffey KW, Roe MT, Dyke CK, et al. Misreporting of myocardial infarction end points: results of adjudication by a central clinical events committee in the PARAGON-B trial. Second Platelet IIb/IIIa Antagonist for the Reduction of Acute Coronary Syndrome Events in a Global Organization Network Trial. Am Heart J 2002; 143(2): 242-8

5. Proposition de règlement du Parlement européen et du Conseil relatif aux essais cliniques de médicaments à usage humain et abrogeant la directive 2001/20/CE. 2012 http://www.senat.fr/europe/textes_europeens/e7534.pdf

6. Strom BL, Kimmel SE, Hennessy S. Pharmacoepidemiology. Fifth edition. Oxford (UK): John Wiley \& Sons 2012

7. European Medicines Agency. Guideline on missing data in confirmatory clinical trials. EMA/CPMP/EWP/1776/99 Rev. 1, 2010 http://www.ema.europa.eu/docs/en_GB/document_library/ Scientific_guideline/2010/09/WC500096793.pdf

8. European Medicines Agency. The European Network of Centres for Pharmacoepidemiology and Pharmacovigilance (ENCePP). Guide on methodological standards in pharmacoepidemiology (Revision 1). 2012 EMA/95098/ 2010. http://www.encepp.eu/standards_and_guidances/documents/ ENCePPGuideofMethStandardsinPE.pdf

9. International Society for Pharmacoepidemiology. Guidelines for Good Pharmacoepidemiology Practices. ISPE. Revision: April 2007 http://www.pharmacoepi.org/resources/guidelines_08027.cfm

10. European Medicines Agency. Guideline on good pharmacovigilance practices (GVP) Module I - Pharmacovigilance systems and their quality systems. EMA/541760/2011. 2012 http://www.ema.europa.eu/docs/en_GB/ document_library/Scientific_guideline/2012/06/WC500129132.pdf

11. European Medicines Agency. ICH Topic E 6 (R1) CPMP/ICH/135/95. EMEA London. Guideline for Good Clinical Practice. 2002 http://www.edctp.org/fileadmin/documents/EMEA_ICHGCP_Guidelines_July_2002.pdf

12. Directive 2001/20/EC. Official Journal of the European Communities, L121/ 34, Luxembourg. 2001 http://eur-lex.europa.eu/LexUriServ/LexUriServ.do?uri=OJ:L:2001:121:0034:0044:en:PDF

13. Ohmann C, Kuchinke W, Canham S, et al. Standard requirements for GCPcompliant data management in multinational clinical trials. Trials 2011; 22(12): 85

14. Hall GC, Sauer B, Bourke A, et al. Guidelines for good database selection and use in pharmacoepidemiology research. Pharmacoepidemiol Drug Saf 2012; 21(1): 1-10

15. GPS - Good practice in secondary data analysis: revision after fundamental reworking. Version 2, January 2008 http://dgepi.de/fileadmin/pdf/leitlinien/gps-version2-final_ENG.pdf

16. The ISPOR good research practices for retrospective database analysis task force report - Part II. Value in Health 2009; 12(8): 1053-61 http://www.ispor.org/TaskForces/documents/RDPartll.pdf 
17. Benchimol EI, Manuel DG, To T, et al. Development and use of reporting guidelines for assessing the quality of validation studies of health administrative data. J Clin Epidemiol 2011; 64(8): 821-9

18. Van Walraven C, Bennett C, Forster AJ. Administrative database research infrequently used validated diagnostic or procedural codes. J Clin Epidemiol 2011; 64(10): 1054-9

19. De Coster C, Quan H, Finlayson A, et al. Identifying prio rities in methodological research using ICD-9-CM and ICD-10 administrative data: report from an international consortium. BMC Health Serv Res 2006; 15 (6): 77
20. Schneeweiss S, Avorn J. A review of uses of health care utilization databases for epidemiologic research on therapeutics. J Clin Epidemiol 2005; 58(4): 323-37

21. Lindquist M. Data quality management in pharmacovigilance. Drug Saf 2004; 27(12): 857-70

Correspondance et offprints: François Alla, Centre d'Épidémiologie clinique, Inserm-CHU de Nancy, Allée du Morvan, 54500 Vandœuvre les Nancy, France.

E-mail : f.alla@chu-nancy.fr 